\title{
Performance of Personality Assessment Inventory and Rorschach Indices of Schizophrenia in a Public Psychiatric Hospital
}

\author{
E. David Klonsky \\ University of Virginia
}

\begin{abstract}
The present study investigated the performance of indices of schizophrenia from the Personality Assessment Inventory (PAI-SCZ; L. C. Morey, 1991) and Rorschach (Rorschach SCZI; J. E. Exner, 1993) in a heterogeneous sample of 24 inpatients at a public psychiatric hospital in the southeastern United States. Results indicated modest agreement between the PAI-SCZ and Rorschach SCZI. More important, the PAI-SCZ but not Rorschach SCZI reliably differentiated inpatients with schizophrenic-spectrum diagnoses from inpatients with other psychiatric diagnoses. In settings in which psychotic disorders falling outside the schizophrenic spectrum are common, the PAI-SCZ may be better suited than the Rorschach SCZI to aid in the differential diagnosis of schizophrenia.
\end{abstract}

Diagnosing schizophrenia can be challenging. There is no chemical or anatomical marker by which a patient can be definitively identified as having schizophrenia (Gottesman, 1991). Instead, clinicians make diagnoses according to the presence of a minimum number of symptoms characteristic of schizophrenia (e.g., delusions, hallucinations, disorganized speech, social/occupational dysfunction) and the absence of other disorders that could account for such symptoms (e.g., mood disorders, medical conditions; American Psychiatric Association, 2001). The absence of a distinct neuropathology requires that a multidimensional assessment of behaviors be conducted to ensure accurate diagnoses (Gottesman, 1991). Even when considering multiple sources of information (e.g., diagnostic interview, behavioral observations, family history), clinicians frequently have difficulty arriving at a diagnosis. Psychological tests may aid in the differential diagnosis between schizophrenia and other disorders found in psychiatric hospitals.

I thank Dennis Donat, Jeffrey Phillips, and Sharon Johnson for their assistance in carrying out this study, and Alexis Black for her comments on earlier versions of this article.

This research was supported in part by National Institute of Mental Health (NIMH) Grant MH51187 and by an NIMH National Research Service Award.

Correspondence concerning this article should be addressed to E. David Klonsky, Department of Psychology, University of Virginia, 102 Gilmer Hall, P.O. Box 400400, Charlottesville, VA 22904-4400. E-mail: klonsky@ virginia.edu
Two tests commonly used in psychiatric hospitals to aid in differential diagnoses are the Personality Assessment Inventory (PAI; Morey, 1991) and the Rorschach Comprehensive System (RCS; Exner, 2000). The PAI is a self-report measure of personality and psychopathology. It includes a schizophrenia scale (SCZ) designed to measure symptoms associated with schizophrenic-spectrum disorders. The PAI-SCZ has repeatedly been demonstrated to correlate with other, well-validated measures of schizophrenic symptomatology (Fantoni-Salvador \& Rogers, 1997; Morey, 1999). In addition, the PAI-SCZ has been shown to differentiate patients with schizophrenia from nonpatient controls (Boyle \& Lennon, 1994). However, it is unclear if the PAI-SCZ can discriminate between diagnostically distinct groups of psychiatric patients. For example, in a study of patients with alcoholism and schizophrenia, the two groups generated similar scores on the PAI-SCZ (Boyle \& Lennon, 1994).

The RCS is a projective measure of personality and psychopathology. The RCS includes a schizophrenia index (SZCI ${ }^{1}$; Exner, 1993) designed to measure the perceptual and cognitive distortions characteristic of patients with schizophrenia and related psychotic disorders. This index correlates minimally with self-report measures of schizophrenic symptom-

${ }^{1}$ With the most recent update of the RCS (Exner, 2000), the SCZI has been revised and renamed the PerceptualThinking Index (PTI). A recent study found that the SCZI and PTI are substantially correlated $(r=.94)$, indicating that the two scales essentially measure the same construct (Smith, Baity, Knowles, \& Hilsenroth, 2001). 
atology such as the Minnesota Multiphasic Personality Inventory (MMPI; Hathaway \& McKinley, 1940; Archer \& Gordon, 1988; Meyer, Riethmiller, Brooks, Benoit, \& Handler, 2000). Nevertheless, the Rorschach SCZI has repeatedly been shown to differentiate patients with schizophrenia and related psychotic disorders from non-psychotic patient groups (Jorgensen, Andersen, \& Dam, 2000). For example, patients with schizophrenia but not patients with severe depression generated elevated scores on the Rorschach SCZI (Ilonen et al., 1999). Likewise, the SCZI differentiated patients with psychotic disorders from patients with personality disorders and participants in a nonclinical control sample (Hilsenroth, Fowler, \& Padawer, 1998).

The studies described above establish the ability of the PAI and RCS schizophrenia indices to distinguish between well-defined groups of patients. ${ }^{2}$ The present study seeks to extend our knowledge of the PAISCZ and Rorschach SCZI by examining how these scales operate in a public psychiatric hospital. In this type of setting, clinicians do not have the benefit of choosing among a predetermined and limited number of diagnostic possibilities. Instead, clinicians in public hospitals make differential diagnoses among the diversity of disorders typically encountered in such settings. The analyses in the current study were conceived with this type of situation in mind. The present study examines the relationship of the Rorschach and PAI schizophrenia indices to each other and to $\mathrm{Di}$ agnostic and Statistical Manual of Mental Disorders (4th ed.; DSM-IV; American Psychiatric Association, 2001) diagnosis in a heterogeneous sample of inpatients at a public psychiatric hospital.

\section{Method}

\section{Participants}

Participants were 24 inpatients (12 men, 12 women) admitted to a public psychiatric hospital in the state of Virginia between 1995 and 2000, who were administered both the PAI and Rorschach. Patients with valid PAI and RCS protocols were identified from an archival search of hospital psychological evaluation files. Participants had a mean age of 35 $(S D=12.4)$. Twenty participants were Caucasian, 3 African American, and 1 Asian.

Diagnoses were made according to DSM-IV criteria by a multidisciplinary treatment team that included a board certified psychiatrist, a licensed clinical psychologist, a licensed clinical social worker, and a registered psychiatric nurse. All team members participated in a comprehensive intake evaluation of symptoms and history. In addition, all team members had the opportunity to interact with the patient as well as to observe the patient's behavior on the ward prior to arriving at a diagnosis. It is important to note that participants' diagnoses were determined by the treatment team before the PAI and Rorschach were administered. Consequently, PAI and Rorschach data did not influence the assignment of diagnoses.

Nine participants were assigned $D S M-I V$ diagnoses of schizophrenic-spectrum disorders (6 schizophrenia, 3 schizoaffective disorder). Diagnoses of the remaining 15 patients included (the number of participants with each diagnosis is indicated in parentheses): bipolar disorder with psychotic features (3), psychotic disorder not otherwise specified (NOS) (2), substance dependence (2), substance-induced psychotic disorder (2), alcohol dependence (1), major depression (1), mood disorder NOS (1), mental disorder due to a medical condition (1), psychological factors affecting a medical condition (1), and delusional disorder (1). Two patients had comorbid Axis II diagnoses of borderline personality disorder.

\section{Measures}

The PAI is a 344-item self-report instrument that requires $50-60 \mathrm{~min}$ to administer. The PAI-SCZ is a 24-item scale designed to assess symptomatology relevant to the broad spectrum of schizophrenic disorders (Morey, 1999). Participants' $t$ scores on the PAISCZ were analyzed in the present study.

The Rorschach is a psychological projective test of personality that requires approximately $96 \mathrm{~min}$ to administer (Ball, Archer, \& Imhof, 1994). The RCS includes the SCZI, designed to aid in the assessment of schizophrenia and related disorders (Exner, 1993). The SCZI is based on form quality, cognitive special scores, and human movement. Scores on the SCZI can range from 0 to 6 . Participants' scores on the SCZI were analyzed in the present study.

\section{Results}

The PAI-SCZ and Rorschach SCZI were positively correlated $(r=.42, p=.04)$. A two-tailed $t$ test revealed that PAI-SCZ $t$ scores were substantially higher for the schizophrenic spectrum sample $(M=77.4, S D=15.5)$ than for the nonschizo-

\footnotetext{
${ }^{2}$ The present literature review of Rorschach validity is by design limited only to the SCZI. The validity of many RCS indices remains controversial (e.g., Lilienfeld, Wood, \& Garb, 2000; Viglione, 1999).
} 
phrenic sample $(M=58.5, S D=13.4), t(22)=$ $3.16, p=.005$. Rorschach SCZI scores were not higher for patients with schizophrenic spectrum disorders $(M=3.11, S D=2.15)$ than for those with other psychiatric disorders $(M=2.93, S D=1.62)$, $t(22)=0.23, p=.82$.

For both indices, optimal cutoff points for assigning a diagnosis of schizophrenia were determined, and overall classification accuracy, sensitivity, specificity, positive predictive power (PPP), and negative predictive power (NPP) were calculated. For the PAISCZ, an optimal cutoff point of $67 T$ accurately classified $79 \%$ of patients. Sensitivity was $78 \%$, specificity $75 \%$, PPP $70 \%$, and NPP $86 \%$. For the Rorschach SCZI, an optimal cutoff point of 4 accurately classified $54 \%$ of participants. Sensitivity was $44 \%$, specificity $60 \%$, PPP 40\%, and NPP 64\%.

\section{Discussion}

In this study, I examined the relationship of the PAI-SCZ and Rorschach SCZI to each other and to $D S M-I V$ diagnosis in a heterogeneous sample of 24 inpatients at a public psychiatric hospital. Results indicate modest but reliable convergence between the PAI and Rorschach schizophrenia indices. This result is somewhat surprising because the relationship between information obtained from Rorschach and selfreport indices is typically small or negligible (Archer \& Krishnamurthy, 1993; Lilienfeld, Wood, \& Garb, 2000). In the limited research on the relationship of the SZCI to self-report measures (i.e., MMPI Scale $8)$, correlations were slightly above $0(r \approx .10$; Archer $\&$ Gordon, 1988; Meyer et al., 2000). The convergence observed in the present study is difficult to interpret, particularly given the absence of other published studies on PAI-Rorschach convergence. Further study should address the possibility that the PAISCZ and RCS SCZI are exceptions to the relationships typically observed between self-report and Rorschach indices.

It was also found that the PAI-SCZ was related to participants' $D S M-I V$ diagnoses. Participants with schizophrenic spectrum disorders had substantially higher PAI-SCZ scores than participants with other diagnoses. Just under $80 \%$ of patients were diagnosed accurately by using an optimal cutoff point. These findings are consistent with previous research supporting the convergent validity of the PAI-SCZ (e.g., Fantoni-Salvador \& Rogers, 1997; Morey, 1999). The present study extends this literature, however, by suggesting that the PAI can aid in the differential diagnosis of patients with schizophrenia from among a heterogeneous group of patients with other disor- ders. This application of the PAI may better approximate how psychological tests are typically used in public psychiatric hospitals.

In contrast, the Rorschach SCZI did not reliably differentiate patients with schizophrenic spectrum diagnoses from those with other diagnoses. Although many studies have shown the ability of the SCZI to differentiate patients with schizophrenia from nonpsychotic patient or control groups (Hilsenroth et al., 1998; Ilonen et al., 1999; Jorgensen et al., 2000), the SCZI is probably less able to distinguish patients with schizophrenia from patients with psychotic disorders falling outside the schizophrenic spectrum. The Rorschach SCZI was designed to measure the perceptual and cognitive distortions characteristic of psychotic disorders including but not limited to schizophrenia (Exner, 2000; Hilsenroth et al., 1998; Jorgensen et al., 2000). Consequently, patients with psychotic disorders falling outside the schizophrenic spectrum may generate scores on the SCZI similar to those of patients with schizophrenic-spectrum disorders. For example, Ilonen et al. (1999) reported that $43 \%$ of a sample of manic patients generated elevated scores on the SCZI. As is typical in public psychiatric hospitals, most participants in the present study who did not have schizophrenic spectrum disorders had disorders associated with psychotic features (e.g., psychotic disorder NOS, substance induced psychotic disorder, delusional disorder, bipolar disorder with psychotic features, severe mood disorders, polysubstance dependence). That these patients generated scores on the Rorschach SCZI similar to those of the patients with schizophrenic-spectrum disorders does not refute the construct validity of the SCZI. However, when the differential diagnosis of schizophrenia from other psychotic disorders is of primary concern, the PAI-SCZ may be better suited to the task than the SCZI.

\section{References}

American Psychiatric Association. (2001). Diagnostic and statistical manual of mental disorders (4th ed. text ed.). Washington, DC: Author.

Archer, R. P., \& Gordon, R. A. (1988). MMPI and Rorschach indices of schizophrenic and depressive diagnoses among adolescent inpatients. Journal of Personality Assessment, 52, 276-287.

Archer, R. P., \& Krishnamurthy, R. (1993). A review of MMPI and Rorschach interrelationships in adult samples. Journal of Personality Assessment, 61, 277-293.

Ball, J. D., Archer, R. P., \& Imhof, E. A. (1994). Time requirements of psychological testing: A survey of practitioners. Journal of Personality Assessment, 63, 239-249. 
Boyle, G. J., \& Lennon, T. J. (1994). Examination of the reliability and validity of the Personality Assessment Inventory. Journal of Psychopathology and Behavioral Assessment, 16, 173-187.

Exner, J. E., Jr. (1993). The Rorschach: A comprehensive system: Vol. 1. Basic foundations (3rd ed.). New York: Wiley.

Exner, J. E., Jr. (2000). A primer for Rorschach interpretation. Asheville, NC: Rorschach Workshops.

Fantoni-Salvador, P., \& Rogers, R. (1997). Spanish versions of the MMPI-2 and PAI: An investigation of concurrent validity with Hispanic patients. Assessment, 4, 29-39.

Gottesman, I. I. (1991). Schizophrenia genesis. New York: Freeman Press.

Hathaway, S. R., \& McKinley, J. C. (1940). A multiphasic personality schedule (Minnesota): I. Construction of the schedule. Journal of Psychology, 10, 249-254.

Hilsenroth, M. J., Fowler, J. C., \& Padawer, J. R. (1998). The Rorschach schizophrenia index (SCZI): An examination of reliability, validity, and diagnostic efficiency. Journal of Personality Assessment, 70, 514-534.

Ilonen, T., Taiminen, T., Karlsson, H., Lauerma, H., Leinonen, K., Walleinius, E., et al. (1999). Diagnostic efficiency of the Rorschach schizophrenia and depression indices in identifying first-episode schizophrenia and severe depression. Psychiatry Research, 87, 183-192.
Jorgensen, K., Andersen, T. J., \& Dam, H. (2000). The diagnostic efficiency of the Rorschach Depression Index and the Schizophrenia Index: A review. Assessment, 7 , 259-280.

Lilienfeld, S. O., Wood, J. M., \& Garb, H. N. (2000). The scientific status of projective techniques. Psychological Science in the Public Interest, 1, 27-66.

Meyer, G. J., Riethmiller, R. J., Brooks, R. D., Benoit, W. A., \& Handler, L. (2000). A replication of Rorschach and MMPI-2 convergent validity. Journal of Personality Assessment, 74, 175-215.

Morey, L. C. (1991). Personality Assessment Inventory: Professional manual. Odessa, FL: Psychological Assessment Resources.

Morey, L. C. (1999). Personality Assessment Inventory. In M. E. Mauish (Ed.), The use of psychological testing for treatment planning and outcomes assessment (2nd ed., pp. 1083-1121). Mahwah, NJ: Erlbaum.

Smith, S. R., Baity, M. R., Knowles, E. S., \& Hilsenroth, M. J. (2001). Assessment of disordered thinking in children and adolescents: The Rorschach PerceptualThinking Index. Journal of Personality Assessment, 77, 447-463.

Viglione, D. J. (1999). A review of recent research addressing the utility of the Rorschach. Psychological Assessment, 11, 251-265.

\section{E-Mail Notification of Your Latest Issue Online!}

Would you like to know when the next issue of your favorite APA journal will be available online? This service is now available to you. Sign up at http://watson.apa.org/ notify/ and you will be notified by e-mail when issues of interest to you become available! 